\title{
MARKOV CHAINS WITH HYBRID REPEATING ROWS - UPPER-HESSENBERG, QUASI-TOEPLITZ STRUCTURE OF THE BLOCK TRANSITION PROBABILITY MATRIX
}

\author{
ALEXANDER DUDIN, ${ }^{* * *}$ Belarusian State University \\ CHESOONG KIM,*** Sangji University \\ VALENTINA KLIMENOK, ${ }^{* * * * *}$ Belarusian State University
}

\begin{abstract}
In this paper we consider discrete-time multidimensional Markov chains having a block transition probability matrix which is the sum of a matrix with repeating block rows and a matrix of upper-Hessenberg, quasi-Toeplitz structure. We derive sufficient conditions for the existence of the stationary distribution, and outline two algorithms for calculating the stationary distribution.
\end{abstract}

Keywords: Multidimensional Markov chain; queueing system; ergodicity; algorithm; censoring

2000 Mathematics Subject Classification: Primary 60K25

Secondary $60 \mathrm{~K} 30$

\section{Problem formulation and motivation}

Multidimensional Markov chains having one denumerable and several finite-state components arise as natural mathematical models of many real-life processes; hence, they have received much attention in the literature. Several special cases of such multidimensional Markov chains having a special structure to the block transition probability matrix are effectively analyzed. Three such cases, namely skip-free to the left, skip-free to the right, and quasi-birthand-death processes, have been extensively studied by Neuts. Chains having the skip-free to the left property were studied as M/G/1-type Markov chains in [15]. Chains having the skipfree to the right property are referred to as G/M/1-type Markov chains and were investigated in [14]. Quasi-birth-and-death processes are the Markov chains having both these properties simultaneously. We refer the reader to [18] for an excellent overview of these types of Markov chains as well as for an overview of some future research work. In addition to the skip-free to the left property or skip-free to the right property, the transition probability matrices of M/G/1-type and G/M/1-type Markov chains have a Toeplitz-like structure. This means that the matrix blocks located on the diagonals of the transition probability matrix are identical, except possibly for some boundary rows or columns. In [11] a skip-free to the left Markov chain was considered where the Toeplitz-like structure exhibits only as a limiting case. The corresponding chain is called an asymptotically quasi-Toeplitz Markov chain.

\footnotetext{
Received 30 July 2007.

* Postal address: Laboratory of Applied Probabilistic Analysis, Department of Applied Mathematics and Computer Sciences, Belarusian State University, 4 Independence Avenue, 220030 Minsk-30, Belarus.

** Email address: dudin@bsu.by

*** Postal address: Department of Industrial Engineering, Sangji University, Wonju, Kangwon, 220-702, Korea. Email address: dowoo@sangji.ac.kr

**** Email address: klimenok@bsu.by
} 
A common feature of transition probability matrices for all the abovementioned Markov chains is that all blocks under the first subdiagonal (for skip-free to the left Markov chains) or over the first off-diagonal (for skip-free to the right Markov chains) are equal to 0. Furthermore, the requirement of having such a sparse structure was avoided in several works; for example, Neuts [16] considered the Markov chain whose transitions to the right were almost the same as in the case of M/G/1-type Markov chains, while transitions to the left were possible to all states with equal probability. In [19] and [20] the authors considered the so-called G/G/1-type Markov chains, which possess a Toeplitz-like structure while not necessarily having zero blocks under the first subdiagonal or above the first off-diagonal. Bini et al. [1] provided a systematic treatment of the theory and algorithms for important families of structured Markov chains, and included a good overview of the current literature.

In this paper we will focus on the class of multidimensional Markov chains with possibly all nonzero blocks in the transition probability matrix.

Let $\xi_{n}=\left\{i_{n}, j_{n}\right\}, n \geq 1$, be an irreducible, aperiodic, homogeneous Markov chain with $i_{n} \in\{0,1,2, \ldots\}$. When $i_{n}=i$, the process $j_{n}$ will take values in a finite set $g_{i}$ of finitedimensional vectors. Note that vectors belonging to the set $g_{i}$ can have different dimensions. But there exists a nonnegative integer $N, N \geq 0$, and a set $g$ of finite-dimensional vectors such that $g_{i}=g$ for $i>N$. Thus, the phase space $\&$ of the Markov chain $\xi_{n}, n \geq 1$, has the form

$$
\S=\left\{(i, j), j \in \mathcal{g}_{i}, i=0,1, \ldots, N ;(i, j), j \in \mathcal{g}, i>N\right\} .
$$

In what follows we denote the number of vectors in the sets $g_{i}$ by $J_{i}, i \geq 0$, and denote by $J$ the number of vectors in the set $g$.

Enumerate the states of the chain $\xi_{n}, n \geq 1$, as follows. Set the states $(i, j)$ in ascending order of component $i$ and then, for fixed $i$, arrange the states $(i, j), j \in \mathcal{g}_{i}$, in any suitable manner, $i \geq 0$. Below, without loss of generality, we assume that the states $(i, j), j \in \mathcal{g}_{i}$, are arranged in lexicographic order.

Let $\boldsymbol{P}_{i, l}$ be the $J_{i} \times J_{l}$ matrices formed by the transition probabilities

$$
p_{(i, j),(l, v)}=\mathrm{P}\left\{i_{n+1}=l, j_{n+1}=v \mid i_{n}=i, j_{n}=j\right\}, \quad j \in g_{i}, v \in g_{l}, i, l \geq 0 .
$$

Let the blocking matrix $\boldsymbol{P}=\left(\boldsymbol{P}_{i, l}\right)_{i, l \geq 0}$ have the following structure:

$$
\boldsymbol{P}=\left(\begin{array}{cccccccc}
\boldsymbol{B}_{0,0} & \boldsymbol{B}_{0,1} & \cdots & \boldsymbol{B}_{0, N-1} & \boldsymbol{B}_{0, N} & \boldsymbol{B}_{0, N+1} & \boldsymbol{B}_{0, N+2} & \cdots \\
\vdots & \vdots & \ddots & \vdots & \vdots & \vdots & \vdots & \ddots \\
\boldsymbol{B}_{N, 0} & \boldsymbol{B}_{N, 1} & \cdots & \boldsymbol{B}_{N, N-1} & \boldsymbol{B}_{N, N} & \boldsymbol{B}_{N, N+1} & \boldsymbol{B}_{N, N+2} & \cdots \\
\boldsymbol{A}_{0} & \boldsymbol{A}_{1} & \cdots & \boldsymbol{A}_{N-1} & \boldsymbol{A}_{N}+\boldsymbol{Y}_{0} & \boldsymbol{A}_{N+1}+\boldsymbol{Y}_{1} & \boldsymbol{A}_{N+2}+\boldsymbol{Y}_{2} & \cdots \\
\boldsymbol{A}_{0} & \boldsymbol{A}_{1} & \cdots & \boldsymbol{A}_{N-1} & \boldsymbol{A}_{N} & \boldsymbol{A}_{N+1}+\boldsymbol{Y}_{0} & \boldsymbol{A}_{N+2}+\boldsymbol{Y}_{1} & \cdots \\
\boldsymbol{A}_{0} & \boldsymbol{A}_{1} & \cdots & \boldsymbol{A}_{N-1} & \boldsymbol{A}_{N} & \boldsymbol{A}_{N+1} & \boldsymbol{A}_{N+2}+\boldsymbol{Y}_{0} & \cdots \\
\vdots & \vdots & \ddots & \vdots & \vdots & \vdots & \vdots & \ddots
\end{array}\right)
$$

where the matrices $\boldsymbol{Y}_{l}, l \geq 0$, are nonnegative.

Let

$$
\boldsymbol{A}(z)=\sum_{j=0}^{\infty} \boldsymbol{A}_{j} z^{j}, \quad Y(z)=\sum_{j=0}^{\infty} \boldsymbol{Y}_{j} z^{j}, \quad|z| \leq 1 .
$$

The matrix $\boldsymbol{P}$ can be represented as a sum of two matrices. One matrix has the same structure as the transition probability matrix of the M/G/1-type Markov chain. The second 
matrix has, except possibly for several boundary states, identical block rows. These kinds of matrices appear in the investigation by Chakravarthy [2] of queueing systems (the MAP/G/1type system in particular) with group service. In these systems service is provided only to groups with the fixed minimal size. All customers present in the system at the service beginning epoch are served simultaneously, and the service time does not depend on the size of a group. This explains why the block rows of the transition probability matrix, except for several boundary rows, are identical. A $\boldsymbol{P}$ matrix of the form (1) describes the transitions of the embedded Markov chain for generalization of the model [2] to the case where, at any service beginning epoch, with some probability, all customers are sent to the service simultaneously or, with supplementary probability, only one customer is picked up for the service. A $\boldsymbol{P}$ matrix of the form (1) also arises as a transition probability matrix of the embedded, at service beginning epochs, Markov chain for the BMAP/SM/1 queue with disasters in the case of noninstantaneous recovery of the server and customer accumulation during the recovery time.

The special case of the Markov chain, which is the subject of our paper, was considered in [4] and [5], where the BMAP/SM/1 queue with a Markovian arrival process of disasters was under study. In this case all matrices $\boldsymbol{A}_{l}, l \geq 1$, are equal to $\mathbf{0}$. Only the $\boldsymbol{A}_{0}$ matrix is nonzero.

Contributions of analysis presented in our paper comparing to [4] and [5] consist of the following.

(i) In contrast to [4] and [5] we do not assume in advance any explicit form for the blocks $\boldsymbol{P}_{i, j}$. Thus, the problem of establishing conditions for ergodicity of the considered Markov chain arises. Such a problem was not considered in [4] and [5] because it was intuitively clear that specifics of the Markov chain always guaranteed ergodicity. In the general case considered in this paper, the solution of this problem is not trivial and assumes an analysis of several possible structures for the matrices $\boldsymbol{A}(1)$ and $\boldsymbol{Y}(1)$.

(ii) While extension of the algorithm based on the transform approach, which was described in [4], to the case of the considered Markov chain looks straightforward, elaboration of a numerically stable algorithm similar to the one considered in [5] is not simple. Derivation of the algorithm in [5] essentially followed the approach introduced by Ramaswami [17] and exploited the fact that the denumerable component of the Markov chain can reach any level $m, m \geq 1$, starting from level $k, k>m$, via only the levels $k-1, \ldots, m+1$. The Markov chain $\xi_{n}, n \geq 1$, considered in this paper does not possess such a property. So, in the present paper the derivation of the algorithm, which is similar to the algorithm used in [5] and [17], is performed by means of preliminary consideration of an auxiliary Markov chain and by further applying the censoring technique.

The remainder of the paper is organized as follows. In Section 2 sufficient conditions for ergodicity of the Markov chain $\xi_{n}, n \geq 1$, are derived. In Section 3 two algorithms used to calculate the stationary distribution of this Markov chain are outlined.

\section{Stability condition}

To obtain the ergodicity condition, we will use the mean drift approach. To this end, we formulate the vector analog of Moustafa's well-known theorem [13]. 
Proposition 1. Suppose that there exist $\varepsilon>0$, a positive integer $i_{0}$, and a set of nonnegative vectors $\boldsymbol{X}_{i}, i \geq 0$ (vector test function), such that the following inequalities are valid:

$$
\begin{gathered}
\sum_{j=0}^{\infty} \boldsymbol{P}_{i, j} \boldsymbol{X}_{j}-\boldsymbol{X}_{i}<-\varepsilon \boldsymbol{e}, \quad i>i_{0}, \\
\sum_{j=0}^{\infty} \boldsymbol{P}_{i, j} \boldsymbol{X}_{j}<\infty, \quad i=\overline{0, i_{0}}
\end{gathered}
$$

where $\boldsymbol{e}$ is a column vector consisting of 1 s and $\overline{0, i_{0}}$ means $i=0,1, \ldots, i_{0}$. Then the Markov chain $\xi_{n}, n \geq 1$, is ergodic.

We distinguish between the cases of an irreducible and a reducible matrix $\boldsymbol{Y}(1)$. Note that in the latter case the matrix generating function $\boldsymbol{Y}(z)$ is also a reducible matrix and has the same structure as $\boldsymbol{Y}(1)$. Without loss of generality, we assume that the matrix $\boldsymbol{Y}(z)$ is already presented in a normal form (for a definition, see [7] and [15]), i.e. it has the following structure:

$$
\boldsymbol{Y}(z)=\left(\begin{array}{ccccccc}
\boldsymbol{Y}^{(1)}(z) & \mathbf{0} & \cdots & \mathbf{0} & \mathbf{0} & \cdots & \mathbf{0} \\
\vdots & \vdots & \ddots & \vdots & \vdots & \ddots & \vdots \\
\mathbf{0} & \mathbf{0} & \cdots & \boldsymbol{Y}^{(m)}(z) & \mathbf{0} & \cdots & \mathbf{0} \\
\boldsymbol{Y}^{(m+1,1)}(z) & \boldsymbol{Y}^{(m+1,2)}(z) & \cdots & \boldsymbol{Y}^{(m+1, m)}(z) & \boldsymbol{Y}^{(m+1)}(z) & \cdots & \mathbf{0} \\
\vdots & \vdots & \ddots & \vdots & \vdots & \ddots & \vdots \\
\boldsymbol{Y}^{(s, 1)}(z) & \boldsymbol{Y}^{(s, 2)}(z) & \cdots & \boldsymbol{Y}^{(s, m)}(z) & \boldsymbol{Y}^{(s, m+1)}(z) & \cdots & \boldsymbol{Y}^{(s)}(z)
\end{array}\right),
$$

where $\boldsymbol{Y}^{(1)}(z), \ldots, \boldsymbol{Y}^{(s)}(z)$ are irreducible square matrices of size $d_{1}, \ldots, d_{s}$, respectively, and there is at least one nonzero matrix in each row $\boldsymbol{Y}^{(n, 1)}(z), \ldots, \boldsymbol{Y}^{(n, n-1)}(z), n=m+1, \ldots, s$.

Lemma 1. The following statements are true.

1. Let $\boldsymbol{Y}(1)$ be an irreducible, substochastic matrix. Then there exists an integer $L_{0}, L_{0} \geq$ $N$ such that the matrices $\boldsymbol{\Omega}_{L}=\sum_{l=L}^{\infty} \boldsymbol{A}_{l}+\boldsymbol{Y}(1), L \geq L_{0}$, are also irreducible, substochastic matrices.

2. Let $\boldsymbol{Y}(1)$ be a reducible matrix of the form (4), and let $\boldsymbol{Y}^{(1)}(1), \ldots, \boldsymbol{Y}^{(m)}(1)$ be substochastic matrices. Then there exists an integer $L_{0}, L_{0} \geq N$, such that, for all $L \geq L_{0}$, either

(i) the matrices $\boldsymbol{\Omega}_{L}$ are irreducible and substochastic; or

(ii) the matrices $\boldsymbol{\Omega}_{L}$ are reducible matrices with normal form

$$
\boldsymbol{\Omega}_{L}=\left(\begin{array}{ccccccc}
\boldsymbol{\Omega}_{L}^{(1)} & \mathbf{0} & \cdots & \mathbf{0} & \mathbf{0} & \cdots & \mathbf{0} \\
\vdots & \vdots & \ddots & \vdots & \vdots & \ddots & \vdots \\
\mathbf{0} & \mathbf{0} & \cdots & \boldsymbol{\Omega}_{L}^{(k)} & \mathbf{0} & \cdots & \mathbf{0} \\
\boldsymbol{\Omega}_{L}^{(k+1,1)} & \boldsymbol{\Omega}_{L}^{(k+1,2)} & \cdots & \boldsymbol{\Omega}_{L}^{(k+1, k)} & \boldsymbol{\Omega}_{L}^{(k+1)} & \ldots & \mathbf{0} \\
\vdots & \vdots & \ddots & \vdots & \vdots & \ddots & \vdots \\
\boldsymbol{\Omega}_{L}^{(t, 1)} & \boldsymbol{\Omega}_{L}^{(t+1,1)} & \cdots & \boldsymbol{\Omega}_{L}^{(t, k)} & \boldsymbol{\Omega}_{L}^{(t, k+1)} & \ldots & \boldsymbol{\Omega}_{L}^{(t)}
\end{array}\right)
$$


where $2 \leq t \leq s, 0 \leq k \leq m$, and the matrices $\boldsymbol{\Omega}_{L}{ }^{(n)}, n=\overline{1, t}$, are irreducible and substochastic.

3. Let $\boldsymbol{Y}(1)$ be a reducible matrix of the form (4), where the matrices $\boldsymbol{Y}^{(r)}(1), r=\overline{1, R}, R \leq$ $m$, are stochastic. Then there exists an integer $L_{0} \geq N$ such that, for any $L \geq L_{0}$, the matrices $\boldsymbol{\Omega}_{L}$ are of the form (5), where $k \geq R$, the matrices $\boldsymbol{\Omega}_{L}{ }^{(r)}, r=\overline{1, R}$, are stochastic, and the matrices $\boldsymbol{\Omega}_{L}{ }^{(r)}, r=\overline{R+1, t}$, are substochastic.

The proof of Lemma 1 is based on a careful analysis of the structure of the matrices $Y(1)$ and $\boldsymbol{\Omega}_{L}$. It is straightforward and so is omitted here.

Theorem 1. Let

$$
\sum_{k=1}^{\infty} k \boldsymbol{B}_{i, i+k-1} \boldsymbol{e}<\infty, \quad i=\overline{0, N}, \quad \boldsymbol{Y}^{\prime}(1)<\infty, \quad \boldsymbol{A}^{\prime}(1)<\infty .
$$

(i) If the matrix $\boldsymbol{Y}(1)$ satisfies condition 1 or condition 2 of Lemma 1 then the Markov chain $\xi_{n}, n \geq 1$, is ergodic.

(ii) If $\boldsymbol{Y}(1)$ is an irreducible, stochastic matrix then a sufficient condition for the ergodicity of the Markov chain $\xi_{n}, n \geq 1$, is that the inequality

$$
[\operatorname{det}(z \boldsymbol{I}-\boldsymbol{Y}(z))]_{z=1}^{\prime}>0,
$$

where I denotes the identity matrix, be fulfilled.

(iii) If $\boldsymbol{Y}(1)$ is a reducible matrix of the form (4) and if there exists a set of indices

$$
\left\{n_{1}, n_{2}, \ldots, n_{R}\right\} \subset\{1,2, \ldots, m\}
$$

such that the matrices $\boldsymbol{Y}^{\left(n_{r}\right)}(1), r=\overline{1, R}, R \leq m$, are stochastic, then the sufficient condition for the ergodicity of the Markov chain $\xi_{n}, n \geq 1$, is that the inequalities

$$
\left[\operatorname{det}\left(z \boldsymbol{I}-\boldsymbol{Y}^{\left(n_{r}\right)}(z)\right)\right]_{z=1}^{\prime}>0, \quad r=\overline{1, R},
$$

be fulfilled.

Proof. Denote the left-hand side of inequality (2) by $\boldsymbol{\Gamma}_{i}$,

$$
\boldsymbol{\Gamma}_{i}=\sum_{j=0}^{\infty} \boldsymbol{P}_{i, j} \boldsymbol{X}_{j}-\boldsymbol{X}_{i}, \quad i>i_{0}
$$

Note that $\boldsymbol{\Gamma}_{i}$ can be considered as a vector of mean drifts of the chain in the states having the value $i$ of the denumerable component.

Take the vector test function $\boldsymbol{X}_{i}, i \geq 0$, of the form

$$
\boldsymbol{X}_{i}= \begin{cases}\mathbf{0}, & i \leq L-1, \\ (i+1) \boldsymbol{e}+\boldsymbol{\alpha}, & i>L-1,\end{cases}
$$

where $\boldsymbol{\alpha}$ is a real-valued column vector which will be defined below, and $L$ is some integer such that $L>L_{0}$, where the quantity $L_{0}$ is as defined in Lemma 1 . 
Setting $i_{0}=L$ and substituting $\boldsymbol{X}_{i}, i \geq 0$, of the form (9) into (8), we derive the following expression for the vectors $\boldsymbol{\Gamma}_{i}$ :

$$
\boldsymbol{\Gamma}_{i}=\sum_{j=L}^{\infty} \boldsymbol{A}_{j}(j+1) \boldsymbol{e}+\left.(\boldsymbol{Y}(z)-z \boldsymbol{I})^{\prime}\right|_{z=1} \boldsymbol{e}+\left(\boldsymbol{\Omega}_{L}-\boldsymbol{I}\right) \boldsymbol{\alpha}, \quad i>L .
$$

The vectors (10) do not depend on $i$. So, in what follows we set $\boldsymbol{\Gamma}_{i}=-\boldsymbol{\Delta}, i>L$.

Let the matrix $\boldsymbol{Y}$ (1) satisfy condition (i) of Theorem 1. Fix the value $L>L_{0}$. Let us show that there exists a positive vector $\boldsymbol{\alpha}$ such that $\boldsymbol{\Delta}>\boldsymbol{0}$.

Let

$$
\boldsymbol{\beta}=\sum_{j=L}^{\infty} \boldsymbol{A}_{j}(j+1) \boldsymbol{e}+\left.(\boldsymbol{Y}(z)-z \boldsymbol{I})^{\prime}\right|_{z=1} \boldsymbol{e}+\boldsymbol{\Delta},
$$

and choose $\boldsymbol{\Delta}$ to be a positive vector whose entries are greater than the corresponding entries of the vector $\left.(\boldsymbol{Y}(z)-z \boldsymbol{I})^{\prime}\right|_{z=1} \boldsymbol{e}$ in modulus. This implies that $\boldsymbol{\beta}>\mathbf{0}$.

Consider the following system of linear algebraic equations for the components of a vector $\alpha$ :

$$
\left(\boldsymbol{I}-\boldsymbol{\Omega}_{L}\right) \boldsymbol{\alpha}=\boldsymbol{\beta} .
$$

By Lemma 1, the matrix $\boldsymbol{\Omega}_{L}$ is either an irreducible, substochastic matrix or a reducible, substochastic matrix of the form (5), where the diagonal blocks $\Omega_{L}{ }^{(n)}, n=\overline{1, t}$, are irreducible, substochastic matrices. So, the matrix $\left(\boldsymbol{I}-\boldsymbol{\Omega}_{L}\right)^{-1}$ exists and is nonnegative. This and the inequality $\boldsymbol{\beta}>\mathbf{0}$ imply that system (11) has the unique nonnegative solution $\boldsymbol{\alpha}=\left(\boldsymbol{I}-\boldsymbol{\Omega}_{L}\right)^{-1} \boldsymbol{\beta}$. Substituting this solution into (9), we define the test function satisfying Moustafa's theorem and guaranteeing the satisfaction of the relations $\boldsymbol{\Gamma}_{i}=-\boldsymbol{\Delta}, i>L$, where $\boldsymbol{\Delta}$ is a positive vector. This means that (2) is satisfied.

It is easy to show that the constructed test function also guarantees the satisfaction of (3). So, the chain $\xi_{n}, n \geq 1$, is ergodic.

If the matrix $\boldsymbol{Y}$ (1) satisfies condition (ii) of Theorem 1 then the chain $\xi_{n}, n \geq 1$, is of M/G/1 type and the statement of the theorem follows from the corresponding theory; see, e.g. [6] and [11].

Now let us assume that matrix $\boldsymbol{Y}$ (1) satisfies condition (iii) of Theorem 1.

Let us show that there exists a positive vector $\boldsymbol{\Delta}$ such that the following system has a solution $\alpha$ :

$$
\left(\boldsymbol{I}-\boldsymbol{\Omega}_{L}\right) \boldsymbol{\alpha}=\sum_{j=L}^{\infty} \boldsymbol{A}_{j}(j+1) \boldsymbol{e}+\left.(\boldsymbol{Y}(z)-z \boldsymbol{I})^{\prime}\right|_{z=1} \boldsymbol{e}+\boldsymbol{\Delta} .
$$

Without loss of generality, we will assume that $n_{r}=r, r=\overline{1, R}$. Then matrix $Y(1)$ satisfies condition 3 of Lemma 1. Therefore, the matrix $\boldsymbol{\Omega}_{L}$ is of the form (5), where $k \geq R$, the matrices $\boldsymbol{\Omega}_{L}{ }^{(r)}, r=\overline{1, R}$, are stochastic, and the matrices $\boldsymbol{\Omega}_{L}{ }^{(r)}, r=\overline{R+1, t}$, are irreducible and substochastic.

Let us partition the vector $\boldsymbol{\alpha}$ as $\boldsymbol{\alpha}=\left(\boldsymbol{\alpha}^{(1)^{\top}}, \ldots, \boldsymbol{\alpha}^{(R)^{\top}}, \boldsymbol{\alpha}^{(R+1)^{\top}}\right)^{\top}$, where $\boldsymbol{\alpha}^{(r)}$ is a column vector of dimension $d_{r}, r=\overline{1, R}$. Correspondingly, partition the vector $\boldsymbol{\Delta}$ as $\boldsymbol{\Delta}=$ $\left(\boldsymbol{\Delta}^{(1)^{\top}}, \ldots, \boldsymbol{\Delta}^{(R)^{\top}}, \boldsymbol{\Delta}^{(R+1)^{\top}}\right)^{\top}$. Then system (12) is decomposed as follows:

$$
\begin{gathered}
\left(\boldsymbol{I}-\boldsymbol{Y}^{(r)}(1)\right) \boldsymbol{\alpha}^{(r)}=\left.\left(\boldsymbol{Y}^{(r)}(z)-z \boldsymbol{I}\right)^{\prime}\right|_{z=1} \boldsymbol{e}+\boldsymbol{\Delta}^{(r)}, \quad r=\overline{1, R}, \\
\left(\hat{\boldsymbol{I}}-\hat{\boldsymbol{\Omega}}_{L}\right) \boldsymbol{\alpha}=\left.\left(\hat{\boldsymbol{\Omega}}_{L}(z)-z \hat{\boldsymbol{I}}\right)^{\prime}\right|_{z=1} \boldsymbol{e}+\boldsymbol{\Delta}^{(R+1)},
\end{gathered}
$$


where $\hat{\boldsymbol{\Omega}}_{L}, \hat{\boldsymbol{\Omega}}_{L}(z)$, and $\hat{\boldsymbol{I}}$ are rectangular matrices formed by the last $J-\sum_{r=1}^{R} d_{r}$ rows of the matrices $\boldsymbol{\Omega}_{L}, \sum_{j=L}^{\infty} \boldsymbol{A}_{j} z^{j+1}+\boldsymbol{Y}(z)$, and $\boldsymbol{I}_{J}$, respectively.

From Lemma 1 of [11] it follows that, under condition (7) and the assumption that $n_{r}=$ $r, r=\overline{1, R}$, there exist positive column vectors $\boldsymbol{\Delta}^{(r)}=\Delta_{0}{ }^{(r)}, r=\overline{1, R}$, such that each system in (13) has an infinite set of solutions. Let $\boldsymbol{\alpha}_{0}{ }^{(r)}, r=\overline{1, R}$, be some solution of (13). Let us substitute this solution into (9) and fix the value $L>L_{0}$, providing the nonnegativity of the corresponding entries of the vectors $\boldsymbol{X}_{i}, i \geq L$. Substituting $\boldsymbol{\alpha}^{(r)}=\boldsymbol{\alpha}_{0}{ }^{(r)}, r=\overline{1, R}$, into (14) we obtain the following system of linear algebraic equations for the components of the vector $\boldsymbol{\alpha}^{(R+1)}$ :

$$
\left(\boldsymbol{I}-\hat{\boldsymbol{\Omega}}_{L}^{(1)}\right) \boldsymbol{\alpha}^{(R+1)}=\hat{\boldsymbol{\Omega}}_{L}^{(2)}\left(\boldsymbol{\alpha}_{0}^{(1)^{\top}}, \ldots, \boldsymbol{\alpha}_{0}^{(R)^{\top}}\right)^{\top}+\left.\left(\hat{\boldsymbol{\Omega}}_{L}(z)-z \hat{\boldsymbol{I}}\right)^{\prime}\right|_{z=1} \boldsymbol{e}+\boldsymbol{\Delta}^{(R+1)},
$$

where the matrix $\hat{\boldsymbol{\Omega}}_{L}^{(1)}$ is formed by the last $J-\sum_{r=1}^{R} d_{r}$ columns of the matrix $\hat{\boldsymbol{\Omega}}_{L}$, and the matrix $\hat{\mathbf{\Omega}}_{L}^{(2)}$ is formed by the first $\sum_{r=1}^{R} d_{r}$ columns of the matrix $\hat{\boldsymbol{\Omega}}_{L}-\hat{\boldsymbol{I}}$.

By construction, the matrix $\hat{\boldsymbol{\Omega}}_{L}^{(1)}$ is either an irreducible, substochastic matrix or a reducible, substochastic matrix having only substochastic matrices as irreducible diagonal blocks. Then the inverse $\left(\boldsymbol{I}-\hat{\boldsymbol{\Omega}}_{L}\right)^{-1}$ exists and is nonnegative. Substitute $\boldsymbol{\Delta}^{(R+1)}=\boldsymbol{\Delta}_{0}{ }^{(R+1)}$ into (15), where the vector $\boldsymbol{\Delta}_{0}(R+1)$ is chosen as a positive vector with entries providing a positivity of the right-hand side of (15). Then (15) has the unique nonnegative solution $\boldsymbol{\alpha}_{0}^{(R+1)}$. Substituting $\boldsymbol{\alpha}=\boldsymbol{\alpha}_{0}$ into (9), we define the test function satisfying Moustafa's theorem and guaranteeing the satisfaction of the relations $\boldsymbol{\Gamma}_{i}=-\boldsymbol{\Delta}_{0}, i>L$, where $\boldsymbol{\Delta}_{0}$ is a positive vector. This means that (2) is satisfied.

It is easy to show that the constructed test function also guarantees the satisfaction of (3). So, the chain $\xi_{n}, n \geq 1$, is ergodic.

Corollary 1. Let the inequalities $\sum_{k=1}^{\infty} k \boldsymbol{B}_{i, i+k-1} \boldsymbol{e}<\infty, i=\overline{0, N}$, and $\boldsymbol{A}^{\prime}(1)<\infty$ be satisfied. If $\boldsymbol{Y}(1)=\mathbf{0}$ then the Markov chain $\xi_{n}, n \geq 1$, is ergodic.

Corollary 2. If the vector $\boldsymbol{A}(1) \boldsymbol{e}$ is positive and inequalities (6) are satisfied then the Markov chain $\xi_{n}, n \geq 1$, is ergodic.

\section{Algorithms for calculating the stationary distribution}

For the sake of simplifying the presentation, we will further assume that the parameter $N$ in (1) is equal to 0 , i.e. the Markov chain $\xi_{n}, n \geq 1$, has irregular behavior only when the state of the denumerable component $i_{n}, n \geq 1$, is equal to 0 , and suppose that the matrices $\boldsymbol{B}_{0, l}, l \geq 0$, can be presented in the form $\boldsymbol{B}_{0, l}=\boldsymbol{A}_{l}+\boldsymbol{V}_{l}, l \geq 0$, where the matrices $\boldsymbol{V}_{l}, l \geq 0$, are nonnegative.

Thus, in the sequel we assume that matrix (1) has the form

$$
\boldsymbol{P}=\left(\begin{array}{cccccc}
\boldsymbol{A}_{0}+\boldsymbol{V}_{0} & \boldsymbol{A}_{1}+\boldsymbol{V}_{1} & \boldsymbol{A}_{2}+\boldsymbol{V}_{2} & \boldsymbol{A}_{3}+\boldsymbol{V}_{3} & \boldsymbol{A}_{4}+\boldsymbol{V}_{4} & \ldots \\
\boldsymbol{A}_{0}+\boldsymbol{Y}_{0} & \boldsymbol{A}_{1}+\boldsymbol{Y}_{1} & \boldsymbol{A}_{2}+\boldsymbol{Y}_{2} & \boldsymbol{A}_{3}+\boldsymbol{Y}_{3} & \boldsymbol{A}_{4}+\boldsymbol{Y}_{4} & \ldots \\
\boldsymbol{A}_{0} & \boldsymbol{A}_{1}+\boldsymbol{Y}_{0} & \boldsymbol{A}_{2}+\boldsymbol{Y}_{1} & \boldsymbol{A}_{3}+\boldsymbol{Y}_{2} & \boldsymbol{A}_{4}+\boldsymbol{Y}_{3} & \ldots \\
\boldsymbol{A}_{0} & \boldsymbol{A}_{1} & \boldsymbol{A}_{2}+\boldsymbol{Y}_{0} & \boldsymbol{A}_{3}+\boldsymbol{Y}_{1} & \boldsymbol{A}_{4}+\boldsymbol{Y}_{2} & \ldots \\
\vdots & \vdots & \vdots & \vdots & \vdots & \ddots
\end{array}\right)
$$

The state space $\delta$ of the chain $\xi_{n}, n \geq 1$, is $\&=\{(i, j), i \geq 0, j \in \mathscr{g}\}$. 
In what follows we assume that the stability conditions defined by Theorem 1 are satisfied and introduce the stationary state probabilities

$$
\pi(i, j)=\lim _{n \rightarrow \infty} \mathrm{P}\left\{i_{n}=i, j_{n}=j\right\}, \quad i \geq 0, j \in \mathcal{g},
$$

and the vectors $\pi_{i}$ that consist of stationary state probabilities corresponding to the value $i, i \geq 0$, of the first component of the chain enumerated in lexicographic order.

The Chapman-Kolmogorov equations for these vectors are evidently given by

$$
\boldsymbol{\pi}_{j}=\boldsymbol{\pi}_{0} \boldsymbol{V}_{j}+\sum_{i=0}^{\infty} \boldsymbol{\pi}_{i} \boldsymbol{A}_{j}+\sum_{i=1}^{j+1} \boldsymbol{\pi}_{i} \boldsymbol{Y}_{j-i+1}, \quad j \geq 0 .
$$

For the case in which $\boldsymbol{Y}(1)=\mathbf{0}$, the matrix $\boldsymbol{A}(1)$ should be stochastic, so the matrix $\boldsymbol{V}(1)=\mathbf{0}$. Because the Markov chain $\xi_{n}, n \geq 1$, is assumed to be irreducible, the relation $\boldsymbol{Y}(1)=\boldsymbol{V}(1)=\mathbf{0}$ implies that the matrix $\boldsymbol{A}(1)$ is irreducible. Then the stationary probability vectors $\boldsymbol{\pi}_{j}, j \geq 0$, are computed trivially as

$$
\boldsymbol{\pi}_{j}=\rho \boldsymbol{A}_{j}, \quad j \geq 0,
$$

where the vector $\rho$ is the solution to the system $\rho=\rho \boldsymbol{A}(1), \boldsymbol{\rho} \boldsymbol{e}=1$.

For the case in which $\boldsymbol{A}(1)=\mathbf{0}$, the Markov chain $\xi_{n}, n \geq 1$, is of M/G/1 type, so the vectors $\boldsymbol{\pi}_{j}, j \geq 0$, can be computed by means of the well-known transform approach (see, e.g. [3] and [6]) or by the matrix analytic approach of Neuts [15].

Now let us assume that $\boldsymbol{A}(1) \neq \mathbf{0}$ and $\boldsymbol{Y}(1) \neq \mathbf{0}$. Below we develop both approaches for calculating the stationary distribution of the considered Markov chain $\xi_{n}, n \geq 1$.

\subsection{Algorithm based on the transform approach}

Let

$$
\begin{aligned}
& \boldsymbol{\Pi}(z)=\sum_{i=0}^{\infty} \boldsymbol{\pi}_{i} z^{i}, \\
& \hat{\boldsymbol{\Pi}}(z)=\sum_{i=1}^{\infty} \boldsymbol{\pi}_{i} z^{i}, \\
& \boldsymbol{V}(z)=\sum_{i=0}^{\infty} \boldsymbol{V}_{i} z^{i},
\end{aligned}
$$

for $|z| \leq 1$, and

$$
S=A(1)+\boldsymbol{Y}(1)
$$

First, consider the case in which the matrix $S$ is irreducible.

Theorem 2. The vector generating function $\Pi(z)$ of the stationary distribution of the Markov chain $\xi_{n}, n \geq 1$, is the unique solution to the matrix functional equation

$$
\boldsymbol{\Pi}(z)(z \boldsymbol{I}-\boldsymbol{Y}(z))=\boldsymbol{\rho} \boldsymbol{A}(z) z+\boldsymbol{\pi}_{0}((\boldsymbol{V}(1)-\boldsymbol{Y}(1)) \boldsymbol{T} \boldsymbol{A}(z) z+z \boldsymbol{V}(z)-\boldsymbol{Y}(z)),
$$

which is analytical in the region $|z|<1$, continuous on the circle $|z|=1$, and satisfies the normalization condition $\boldsymbol{\Pi}(1) \boldsymbol{e}=1$, where $\boldsymbol{T}=(\boldsymbol{I}-\boldsymbol{S}+\boldsymbol{e} \boldsymbol{\rho})^{-1}$ and $\boldsymbol{\rho}$ is the left stochastic row 
vector of the matrix $S: \rho=\rho S, \rho e=1$. The vector $\pi_{0}$ is the unique solution to the following system of linear algebraic equations:

$$
\begin{aligned}
& \left.\frac{\mathrm{d}^{n}}{\mathrm{~d} z^{n}}\left(\left(\boldsymbol{\rho} \boldsymbol{A}(z) z+\boldsymbol{\pi}_{0}((\boldsymbol{V}(1)-\boldsymbol{Y}(1)) \boldsymbol{T} \boldsymbol{A}(z) z+z \boldsymbol{V}(z)-\boldsymbol{Y}(z))\right) \operatorname{adj}(z \boldsymbol{I}-\boldsymbol{Y}(z))\right)\right|_{z=z_{k}} \\
& \quad=\mathbf{0}, \quad n=\overline{0, n_{k}-1},
\end{aligned}
$$

where $z_{k}$ is a root of multiplicity $n_{k}$ of the equation

$$
\operatorname{det}(z \boldsymbol{I}-\boldsymbol{Y}(z))=0
$$

in the disc $|z| \leq 1, \operatorname{adj}(z \boldsymbol{I}-\boldsymbol{Y}(z))$ denotes the adjoint matrix of $(z \boldsymbol{I}-\boldsymbol{Y}(z))$, and $\sum n_{k}=J$.

Proof. By multiplying (17) by the corresponding degrees of $z$ and summing, we obtain the matrix functional equation

$$
\boldsymbol{\Pi}(z)(z \boldsymbol{I}-\boldsymbol{Y}(z))=\boldsymbol{\Pi}(1) \boldsymbol{A}(z) z+\boldsymbol{\pi}_{0}(z \boldsymbol{V}(z)-\boldsymbol{Y}(z)) .
$$

Uniqueness of the solution to (21), which is analytical in the region $|z|<1$, continuous on its boundary, and satisfies the normalization condition $\Pi(1) \boldsymbol{e}=1$, follows from the following reasonings. Let there exist another vector function $\boldsymbol{P}(z)$ which satisfies (21), is analytical in the region $|z|<1$, continuous on its boundary, and satisfies the normalization condition $\boldsymbol{P}(1) \boldsymbol{e}=1$. Coefficients $\boldsymbol{p}_{i}, i \geq 0$, of the expansion $\boldsymbol{P}(z)=\sum_{i=0}^{\infty} \boldsymbol{p}_{i} z^{i}$ should satisfy the equilibrium equations, (17). However, it is well known that under the satisfaction of the ergodicity condition, system (17) has a unique solution satisfying the normalization condition. So $\boldsymbol{\pi}_{i}=\boldsymbol{p}_{i}, i \geq 0$, which implies that $\Pi(z)=\boldsymbol{P}(z)$.

Taking $z=1$ in (21), we obtain the relation

$$
\Pi(1)(\boldsymbol{I}-\boldsymbol{S})=\boldsymbol{\pi}_{0}(\boldsymbol{V}(1)-\boldsymbol{Y}(1)) .
$$

Matrix $\boldsymbol{S}$ is stochastic, so the matrix $\boldsymbol{I}-\boldsymbol{S}$ is singular and it is not possible to eliminate the vector $\Pi(1)$ from (22) directly. However, it is known that if the matrix $S$ is irreducible then the matrix $\boldsymbol{I}-\boldsymbol{S}+\boldsymbol{e} \boldsymbol{\rho}$ is nonsingular. So, the relation

$$
\Pi(1)=\rho+\pi_{0}(\boldsymbol{V}(1)-\boldsymbol{Y}(1)) \boldsymbol{T}
$$

follows directly from (22). By substituting (23) into (21) we obtain (18).

System (19) has a solution because otherwise the vector generating function $\Pi(z)$ is unbounded at the points $z_{k}$. This contradicts the analyticity of this function in the region $|z|<1$ and its continuity on the boundary $|z|=1$. The existence of more than one solution to system (19) contradicts the uniqueness of the stationary distribution defined by system (17). This completes the proof.

The existence of $J$ roots of (20) in the region $|z| \leq 1$ can be proved by analogy with [3], [6], and [10].

Now, let us consider the case in which the matrix $S$ is reducible but the matrix $\hat{S}=A(1)+$ $\boldsymbol{V}(1)$ is irreducible.

Theorem 3. The vector generating function $\hat{\mathbf{\Pi}}(z)$ of the stationary distribution of the Markov chain $\xi_{n}, n \geq 1$, is the unique solution to the matrix functional equation

$\hat{\boldsymbol{\Pi}}(z)(z \boldsymbol{I}-\boldsymbol{Y}(z))=\hat{\boldsymbol{\rho}} z(\boldsymbol{A}(z)+\boldsymbol{V}(z)-\boldsymbol{I})+z \hat{\boldsymbol{\Pi}}(1)(\boldsymbol{A}(z)-(\boldsymbol{I}-\boldsymbol{S}+\boldsymbol{e} \hat{\boldsymbol{\rho}}) \hat{\boldsymbol{T}}(\boldsymbol{A}(z)+\boldsymbol{V}(z)-\boldsymbol{I}))$, where $\hat{\boldsymbol{T}}=(\boldsymbol{I}-\hat{\boldsymbol{S}}+\boldsymbol{e} \hat{\boldsymbol{\rho}})^{-1}$ and $\hat{\boldsymbol{\rho}}$ is the left stochastic row vector of the matrix $\hat{\boldsymbol{S}}: \hat{\boldsymbol{\rho}}=$ $\hat{\rho} S, \hat{\rho} e=1$. 
The vector $\hat{\mathbf{\Pi}}(1)$ is the unique solution to the following system of linear algebraic equations:

$$
\begin{aligned}
& \frac{\mathrm{d}^{n}}{\mathrm{~d} z^{n}} z((\hat{\boldsymbol{\rho}}(\boldsymbol{A}(z)+\boldsymbol{V}(z)-\boldsymbol{I})+\hat{\boldsymbol{\Pi}}(1)(\boldsymbol{A}(z)-(\boldsymbol{I}-\boldsymbol{S}+\boldsymbol{e} \hat{\boldsymbol{\rho}}) \hat{\boldsymbol{T}}(\boldsymbol{A}(z)+\boldsymbol{V}(z)-\boldsymbol{I}))) \\
& \times \operatorname{adj}(z \boldsymbol{I}-\boldsymbol{Y}(z)))\left.\right|_{z=z_{k}}=\mathbf{0}, \quad n=\overline{0, n_{k}-1},
\end{aligned}
$$

where $z_{k}$ is a root of multiplicity $n_{k}$ of (20) in the disc $|z| \leq 1$ and $\sum n_{k}=J$.

The vector $\pi_{0}$ is computed using $\pi_{0}=\hat{\boldsymbol{\rho}}-\hat{\boldsymbol{\Pi}}(1)(\boldsymbol{I}-\boldsymbol{S}+\boldsymbol{e} \hat{\boldsymbol{\rho}}) \hat{\boldsymbol{T}}$, and the vector $\boldsymbol{\Pi}(1)$ is computed using $\Pi(1)=\hat{\Pi}(1)+\pi_{0}$.

The proof of Theorem 3 is analogous to the proof of Theorem 2.

Once the vectors $\pi_{0}$ and $\Pi(1)$ have been computed, the rest of the vectors can be computed recursively using

$$
\boldsymbol{\pi}_{j+1}=\left(\boldsymbol{\pi}_{j}-\boldsymbol{\pi}_{0} \boldsymbol{V}_{j}-\boldsymbol{\Pi}(1) \boldsymbol{A}_{j}-\sum_{i=1}^{j} \boldsymbol{\pi}_{i} \boldsymbol{Y}_{j-i+1}\right) \boldsymbol{Y}_{0}^{-1}, \quad j \geq 0,
$$

conditional on the matrix $\boldsymbol{Y}_{0}$ being nonsingular.

\subsection{Algorithm based on the censoring technique}

The algorithm described in Subsection 3.1 has several known disadvantages. Theoretically, the trivial problem of finding the known number of roots of (20) in the unit disc of the complex plane and their multiplicities becomes difficult computationally, especially if they are close roots and the dimension $J$ of the vector $\pi_{0}$ is high. Calculation of the derivatives in (19) can require additional difficult analytical work. Selection of the linearly independent equations in system (19) is also a nontrivial task.

If the vector $\pi_{0}$ is found from system (19) then the factorial moments of the distribution $\boldsymbol{\pi}_{i}, i \geq 0$, can be computed based on (18). However, if the probabilities $\boldsymbol{\pi}_{i}, i>0$, are of interest, recursive calculation by (24) requires the nonsingularity of matrix $\boldsymbol{Y}_{0}$, which is not always true. In addition, recursion (24) is not always numerically stable, owing to the presence of multiple subtraction operations. Thus, an alternative algorithm for computing the probabilities $\boldsymbol{\pi}_{i}, i \geq 0$, which is based on probabilistic reasonings and can be traced back to the algorithm offered for M/G/1-type Markov chains in [17], will be developed in this subsection.

Direct translation of Ramaswami's recursion to the considered class of Markov chains is not possible because they do not possess the skip-free to the left property. All blocks of the transition probability matrix can be nonzero. To overcome this difficulty to some extent, let us introduce the auxiliary multidimensional Markov chain $\eta_{n}, n \geq 1$, having a transition probability matrix of almost M/G/1-type structure, except for the presence of the first nonzero block column and an additional boundary block row. This Markov chain has state space $\{*, 0,1,2, \ldots\}$ of denumerable component and block transition probability matrix of the form

$$
\boldsymbol{P}^{*}=\left(\boldsymbol{P}_{i, l}^{*}\right)_{i, l \in\{*, 0,1,2, \ldots\}}=\left(\begin{array}{ccccccc}
\mathbf{0} & \hat{\boldsymbol{A}}_{0} & \hat{\boldsymbol{A}}_{1} & \hat{\boldsymbol{A}}_{2} & \hat{\boldsymbol{A}}_{3} & \hat{\boldsymbol{A}}_{4} & \cdots \\
\hat{\boldsymbol{A}} & \boldsymbol{V}_{0} & \boldsymbol{V}_{1} & \boldsymbol{V}_{2} & \boldsymbol{V}_{3} & \boldsymbol{V}_{4} & \ldots \\
\hat{\boldsymbol{A}} & \boldsymbol{Y}_{0} & \boldsymbol{Y}_{1} & \boldsymbol{Y}_{2} & \boldsymbol{Y}_{3} & \boldsymbol{Y}_{4} & \ldots \\
\hat{\boldsymbol{A}} & \mathbf{0} & \boldsymbol{Y}_{0} & \boldsymbol{Y}_{1} & \boldsymbol{Y}_{2} & \boldsymbol{Y}_{3} & \ldots \\
\hat{\boldsymbol{A}} & \mathbf{0} & \mathbf{0} & \boldsymbol{Y}_{0} & \boldsymbol{Y}_{1} & \boldsymbol{Y}_{2} & \ldots \\
\vdots & \vdots & \vdots & \vdots & \vdots & \vdots & \ddots
\end{array}\right),
$$


where the nonegative matrices $\boldsymbol{Y}_{l}$ and $\boldsymbol{V}_{l}, l \geq 0$, are defined as above and the matrices $\hat{\boldsymbol{A}}$ and $\hat{\boldsymbol{A}}_{l}, l \geq 0$, are defined as follows.

Consider the diagonal matrix $\bar{A}=\operatorname{diag}\{\boldsymbol{A}(1) \boldsymbol{e}\}$ with diagonal entries defined by the entries of the vector $\boldsymbol{A}(1) \boldsymbol{e}$. The matrix $\hat{\boldsymbol{A}}$ is obtained from the matrix $\overline{\boldsymbol{A}}$ by deleting the columns, if any, with all zero entries. The matrix $\hat{\boldsymbol{A}}_{l}$ is obtained from the matrix $\boldsymbol{A}_{l}, l \geq 0$, by deleting the rows consisting of all zero entries and by dividing the entries of the remaining rows by the corresponding diagonal entry of the matrix $\tilde{\boldsymbol{A}}$.

It can be verified that the Markov chain $\eta_{n}, n \geq 1$, is irreducible and aperiodic and that the matrix $\boldsymbol{P}^{*}$ is of the form (1), where $N=1, \boldsymbol{B}_{0,0}=0, \boldsymbol{B}_{0, j}=\hat{\boldsymbol{A}}_{j}, j \geq 1 ; \boldsymbol{B}_{1,0}=\hat{\boldsymbol{A}}$, $\boldsymbol{B}_{1, j+1}=\boldsymbol{V}_{j}, j \geq 0$; and $\boldsymbol{A}_{0}=\hat{\boldsymbol{A}}, \boldsymbol{A}_{j}=0, j \geq 1$. From this matching, it is easy to see that the sufficient ergodicity conditions given in Theorem 1 for the auxiliary Markov chain $\eta_{n}, n \geq 1$, coincide with the sufficient ergodicity conditions for the original Markov chain $\xi_{n}, n \geq 1$.

Let us consider the censored Markov chain $\zeta_{n}, n \geq 1$, which is obtained from the Markov chain $\eta_{n}, n \geq 1$, by eliminating the states having value $*$ of denumerable component. It has transition probability matrix $\boldsymbol{P}$ of the form (16). It can be verified that the censored Markov chain $\zeta_{n}, n \geq 1$, has the same state space and transition probability matrix as the Markov chain $\xi_{n}, n \geq 1$, which is currently under study. So, their stationary distributions coincide.

There exists a well-known relation (see, e.g. [8] and [9]) between the stationary probability vectors of the censored Markov chains and the original Markov chains. So, if we find the stationary distribution of the Markov chain $\eta_{n}, n \geq 1$, we will be able to compute the stationary distribution of the censored Markov chain $\zeta_{n}, n \geq 1$, with respect to it, and then we will be able to compute the stationary distribution of the original Markov chain $\xi_{n}, n \geq 1$.

This explains the motivation for introducing the auxiliary Markov chain $\eta_{n}, n \geq 1$ : to reduce the investigation of the Markov chain $\xi_{n}, n \geq 1$, with potentially a fully populated transition probability matrix of the form (16), to the consideration of the Markov chain with a more sparse transition probability matrix $\boldsymbol{P}^{*}$.

Let us denote by $\boldsymbol{G}$ the matrix which is the minimal nonnegative solution to the equation

$$
\boldsymbol{G}=\sum_{l=0}^{\infty} \boldsymbol{Y}_{l} \boldsymbol{G}^{l}
$$

The entries of the matrix $\boldsymbol{G}$, which is the straightforward analog of the $\boldsymbol{G}$ matrix introduced by Neuts [15] for M/G/1-type Markov chains, characterize the transition probability of component $j_{n}$ of the Markov chain $\eta_{n}, n \geq 1$, in the time interval during which component $i_{n}$, starting from the state $k+1$, reaches state $k, k \geq 0$, for the first time, without visiting state $*$. It is easy to verify that matrix $\boldsymbol{G}$ does not depend on the value $k, k \geq 0$, and satisfies matrix equation (25). For more details about the existence of the solution to (25) and for some algorithms for solving equations of such type, see, e.g. [15].

The following assertion gives a constructive algorithmic way for computing the stationary distribution of the Markov chain $\eta_{n}, n \geq 1$.

Theorem 4. If $\boldsymbol{Y}(1)$ is an irreducible, substochastic matrix or if $\boldsymbol{Y}(1)$ is a reducible matrix of the form (4) and $\boldsymbol{Y}^{(1)}(1), \ldots, \boldsymbol{Y}^{(m)}(1)$ are substochastic matrices, then the stationary distribution $\boldsymbol{p}_{i}, i \in\{*, 0,1,2, \ldots\}$, of the Markov chain $\eta_{n}, n \geq 1$, can be computed in the following algorithmic way. 
- Calculate the matrices $\boldsymbol{H}$ and $\boldsymbol{H}_{0}$ using the following formulae:

$$
\begin{gathered}
\boldsymbol{H}=(\boldsymbol{I}-\boldsymbol{G})(\boldsymbol{I}-\boldsymbol{Y}(1))^{-1} \hat{\boldsymbol{A}} \\
\boldsymbol{H}_{0}=(\boldsymbol{I}-\boldsymbol{V}(\boldsymbol{G}))^{-1}\left(\hat{\boldsymbol{A}}+\sum_{l=0}^{\infty} \boldsymbol{V}_{l} \sum_{i=0}^{l-1} \boldsymbol{G}^{i} \boldsymbol{H}\right) .
\end{gathered}
$$

- Calculate the matrices $\hat{\boldsymbol{P}}_{l, k}, l, k \in\{*, 0,1,2, \ldots\}$, using

$$
\begin{gathered}
\hat{\boldsymbol{P}}_{0, k}=\sum_{i=0}^{\infty} \boldsymbol{V}_{k+i} \boldsymbol{G}^{i}, \quad k \geq 0, \\
\hat{\boldsymbol{P}}_{n, k}=\sum_{i=0}^{\infty} \boldsymbol{Y}_{k+i-n+1} \boldsymbol{G}^{i}, \quad n=\overline{1, k}, k \geq 1, \\
\hat{\boldsymbol{P}}_{*, k}=\sum_{i=0}^{\infty} \hat{\boldsymbol{A}}_{k+i} \boldsymbol{G}^{i}, \quad k \geq 0, \\
\hat{\boldsymbol{P}}_{*, *}=\sum_{l=0}^{\infty} \hat{\boldsymbol{A}}_{l}\left(\boldsymbol{G}^{l} \boldsymbol{H}_{0}+\sum_{i=0}^{l-1} \boldsymbol{G}^{i} \boldsymbol{H}\right) .
\end{gathered}
$$

- Calculate the matrices $\boldsymbol{\Phi}_{l}, l \geq 0$, from the recursion

$$
\boldsymbol{\Phi}_{l}=\left(\hat{\boldsymbol{P}}_{*, l}+\sum_{n=0}^{l-1} \boldsymbol{\Phi}_{n} \hat{\boldsymbol{P}}_{n, l}\right)\left(\boldsymbol{I}-\hat{\boldsymbol{P}}_{l, l}\right)^{-1}, \quad l \geq 0 .
$$

- Calculate the vector $\boldsymbol{p}_{*}$ as the unique solution to the system

$$
\boldsymbol{p}_{*}\left(\boldsymbol{I}-\hat{\boldsymbol{P}}_{*, *}\right)=\mathbf{0}, \quad \boldsymbol{p}_{*}\left(\boldsymbol{e}+\sum_{l=0}^{\infty} \boldsymbol{\Phi}_{l} \boldsymbol{e}\right)=1 .
$$

- Calculate the vectors $\boldsymbol{p}_{i}, i \geq 0$, using

$$
\boldsymbol{p}_{i}=\boldsymbol{p}_{*} \boldsymbol{\Phi}_{i}, \quad i \geq 0 .
$$

Proof. The entries of matrix $\boldsymbol{H}$ characterize the transition probability of component $j_{n}$ of the Markov chain $\eta_{n}, n \geq 1$, in the time interval during which component $i_{n}$ of this Markov chain, starting from state $k$, reaches state $*$ without visiting state $k-1, k \geq 1$. This matrix does not depend on $k$ and is defined by the equation

$$
\boldsymbol{H}=\hat{\boldsymbol{A}}+\sum_{l=0}^{\infty} \boldsymbol{Y}_{l+1} \sum_{i=0}^{l} \boldsymbol{G}^{i} \boldsymbol{H},
$$

from which (26) evidently follows.

The entries of matrix $\boldsymbol{H}_{0}$ characterize the transition probability of component $j_{n}$ of the Markov chain $\eta_{n}, n \geq 1$, in the time interval during which component $i_{n}$ of this Markov chain, starting from state 0 , reaches state $*$ for the first time. This matrix is defined by the equation

$$
\boldsymbol{H}_{0}=\hat{\boldsymbol{A}}+\sum_{l=0}^{\infty} \boldsymbol{V}_{l}\left(\sum_{i=0}^{l-1} \boldsymbol{G}^{i} \boldsymbol{H}+\boldsymbol{G}^{l} \boldsymbol{H}_{0}\right)
$$

from which (27) evidently follows. 
Matrices $\hat{\boldsymbol{P}}_{m, k}, m=*, 1,2, \ldots$, given by formulae (28)-(31) define the transition probabilities of the censored Markov chain $\eta_{n}^{(k)}, n \geq 1$, which is obtained from the Markov chain $\eta_{n}, n \geq 1$, by censoring on level $k$, i.e. considering its transitions only in the set $\{*, 0,1,2, \ldots, k\}, k=*, 1,2, \ldots$ It is well known that (see, e.g. [8] and [9]) the stationary probability vector of this censored Markov chain is the stationary probability vector of the Markov chain $\eta_{n}, n \geq 1$, restricted to the states $\{*, 0,1, \ldots, k\}$ and normalized to have sum 1 . Thus, the stationary probability vectors $\boldsymbol{p}_{i}, i \in\{*, 0,1,2, \ldots\}$, of the Markov chain $\eta_{n}, n \geq 1$, satisfy the system

$$
\boldsymbol{p}_{k}=\boldsymbol{p}_{*} \hat{\boldsymbol{P}}_{*, k}+\sum_{n=0}^{k} \boldsymbol{p}_{n} \hat{\boldsymbol{P}}_{n, k}, \quad k \geq 0, \boldsymbol{p}_{*}=\boldsymbol{p}_{*} \hat{\boldsymbol{P}}_{*, *} .
$$

Now relations (32)-(34) evidently stem from (35) and the normalization condition. This completes the proof.

Remark 1. We could avoid presenting the vectors $\boldsymbol{p}_{i}, i \geq 0$, in multiplicative form, (34), and use the recursion

$$
\boldsymbol{p}_{l}=\left(\boldsymbol{p}_{*} \hat{\boldsymbol{P}}_{*, l}+\sum_{n=0}^{l-1} \boldsymbol{p}_{n} \hat{\boldsymbol{P}}_{n, l}\right)\left(\boldsymbol{I}-\hat{\boldsymbol{P}}_{l, l}\right)^{-1}, \quad l \geq 0,
$$

for these vectors instead of recursion (32) for the matrices $\boldsymbol{\Phi}_{i}, i \geq 0$. This may reduce the computational cost to some extent; see [12]. However, we prefer to use the multiplicative form, (34), because this essentially simplifies the derivation of the inhomogeneous equation in system (33) for the entries of the vector $\boldsymbol{p}_{*}$.

Now let us consider the case in which $\boldsymbol{Y}(1)$ is a reducible matrix of the form (4), and let some of the matrices $\boldsymbol{Y}^{(1)}(1), \ldots, \boldsymbol{Y}^{(m)}(1)$ be stochastic. Without loss of generality, let us assume that the matrices $\boldsymbol{Y}^{(r)}(1), r=\overline{1, R}, R \leq m$, are stochastic. Let us denote by $d$ the total number of rows in these matrices. Above we denoted by $g$ the phase space of component $j_{n}$ of the Markov chain $\xi_{n}, n \geq 1$, and agreed to enumerate the states of this vector component in lexicographic order. Let us denote by $\tilde{g}$ the subset of the first $d$ vectors in the set $g$. In this case the matrix $(\boldsymbol{I}-\boldsymbol{Y}(1))^{-1}$ in (26) used for calculating matrix $\boldsymbol{H}$ does not exist. So, we have to derive another formula for calculating matrix $\boldsymbol{H}$. Since in the considered case the first $d$ rows of the matrix $\boldsymbol{Y}(1)$ have row sum equal to 1 , correspondingly, the first $d$ rows of the matrix $\hat{\boldsymbol{A}}$ consist of zero entries. This implies that, starting in state $(k, j), k \geq 1, j \in \tilde{\tilde{g}}$, the Markov chain under consideration cannot reach the state with value $*$ for the first component of the Markov chain without visiting the states with value $k-1$ for this component. Thus, we conclude that the first $d$ rows of matrix $\boldsymbol{H}$ consist of zero entries and that this matrix has the following structure:

$$
H=\left(\begin{array}{c}
\mathbf{0} \\
\tilde{\boldsymbol{H}}
\end{array}\right)
$$

where $\mathbf{0}$ is the zero matrix of size $d \times \hat{a}, \tilde{\boldsymbol{H}}$ is the matrix of size $(J-d) \times \hat{a}$, and $\hat{a}$ is the number of columns in matrix $\hat{\boldsymbol{A}}$.

Let us calculate the unknown matrix $\tilde{\boldsymbol{H}}$. To this end, we introduce the following notation. Let $\tilde{\boldsymbol{G}}, \tilde{\boldsymbol{Y}}_{k}, k \geq 0$, and $\tilde{\boldsymbol{Y}}(1)$ be matrices formed by the last $J-d$ rows and columns of the $\boldsymbol{G}$, $\boldsymbol{Y}_{k}, k \geq 0$, and $\boldsymbol{Y}(1)$ matrices, respectively, and let $\tilde{\boldsymbol{A}}$ be the matrix formed by the last $J-d$ 
rows of matrix $\hat{\boldsymbol{A}}$. Using probabilistic reasonings, we derive the following equation for the matrix $\tilde{\boldsymbol{H}}$ :

$$
\tilde{\boldsymbol{H}}=\tilde{\boldsymbol{A}}+\sum_{l=0}^{\infty} \tilde{\boldsymbol{Y}}_{l+1} \sum_{i=0}^{l} \tilde{\boldsymbol{G}}^{i} \tilde{\boldsymbol{H}} .
$$

By construction, the matrix $\tilde{\boldsymbol{Y}}(1)$ is either an irreducible, substochastic matrix or a reducible matrix without stochastic blocks. So, the matrix $\boldsymbol{I}-\tilde{\boldsymbol{Y}}(1)$ is nonsingular and the solution to (37) is given by

$$
\tilde{\boldsymbol{H}}=(\boldsymbol{I}-\tilde{\boldsymbol{G}})(\boldsymbol{I}-\tilde{\boldsymbol{Y}}(1))^{-1} \tilde{\boldsymbol{A}} .
$$

Thus, the following statement holds true.

Theorem 5. Let $\boldsymbol{Y}(1)$ be a reducible matrix of the form (4), and let some of the matrices $\boldsymbol{Y}^{(1)}(1), \ldots, \boldsymbol{Y}^{(m)}(1)$ be stochastic. Then the stationary distribution $\boldsymbol{p}_{i}, i \in\{*, 0,1,2, \ldots\}$, of the Markov chain $\eta_{n}, n \geq 1$, can be computed in the same way as is given in Theorem 4 with the only modification being that matrix $\boldsymbol{H}$ is computed using (36) and (38) instead of (26).

Note that the inverse matrices in (27) and (32) exist. Otherwise, it can be shown that the Markov chain $\eta_{n}, n \geq 1$, is reducible. In addition, these matrices are nonnegative. Thus, the algorithms for calculating the vectors $\boldsymbol{p}_{i}, i \in\{*, 0,1,2, \ldots$,$\} , given by Theorems 4$ and 5 do not include subtraction operations and so they are numerically stable.

Corollary 3. The stationary distribution $\pi_{i}, i \geq 0$, of the Markov chain $\xi_{n}, n \geq 1$, is computed using

$$
\boldsymbol{\pi}_{i}=\frac{\boldsymbol{p}_{i}}{1-\boldsymbol{p}_{*} \boldsymbol{e}}, \quad i \geq 0
$$

The proof follows from the abovementioned relation between the stationary distributions of the auxiliary Markov chain $\eta_{n}, n \geq 1$, the censored Markov chain $\zeta_{n}, n \geq 1$, with respect to it, and the Markov chain $\xi_{n}, n \geq 1$, under study.

\section{Conclusion}

In this paper a new class of structured multidimensional Markov chains has been introduced and investigated. This class includes as partial cases well-known M/G/1-type Markov chains and Markov chains having a transition probability matrix consisting of identical block rows. Such Markov chains arise, for example, in the description of queueing systems with exhaustive group service, and technical and biological systems with disasters and mass migration. The ergodicity condition has been derived for different structures for the blocks of the transition probability matrix. Two algorithms for the computation of the steady state distribution have been derived.

\section{Acknowledgement}

This research was supported by the Korea Research Foundation Grant funded by the Korean Government (MOEHRD) (KRF-2006-613-C00001).

\section{References}

[1] Bini, D. A., Latouche, G. And Meini, B. (2005). Numerical Methods for Structured Markov Chains. Oxford University Press.

[2] Chakravarthy, S. (1993). Analysis of a finite MAP/G/1 queue with group services. Queueing Systems 13, 385-407. 
[3] Dudin, A. N. and Klimenok, V. I. (1999). Multi-dimensional quasi-Toeplitz Markov chains. J. Appl. Math. Stoch. Anal. 12, 393-415.

[4] Dudin, A. N. AND Nishimura, S. (1999). A BMAP/SM/1 queueing system with Markovian arrival of disasters. J. Appl. Prob. 36, 868-881.

[5] Dudin, A. N. and Semenova, O. V. (2004). Stable algorithm for stationary distribution calculation for a BMAP/SM/1 queueing system with Markovian input of disasters. J. Appl. Prob. 42, 547-556.

[6] Gail, H. R., Hantler, S. L. And Taylor, B. A. (1996). Spectral analysis of M/G/1 and G/M/1 type Markov chains. Adv. Appl. Prob. 28, 114-165.

[7] Gantmakher, F. R. (1967). The Matrix Theory. Science, Moscow.

[8] Grassmann, W. K. and Heyman, D. P. (1990). Equilibrium distribution of block-structured Markov chains with repeating rows. J. Appl. Prob. 27, 557-576.

[9] Kemeni, J. G., Snell, J. L. And Knapp, A. W. (1966). Denumerable Markov Chains. Van Nostrand, New York.

[10] KlimenoK, V. I. (2001). On the modification of Rouche's theorem for queueing theory problems. Queueing Systems 38, 431-434.

[11] KlimenoK, V. I. And Dudin, A. N. (2006). Multi-dimensional asymptotically quasi-Toeplitz Markov chains and their application in queueing theory. Queueing Systems 54, 245-259.

[12] Meini, B. (1997). An improved FFT-based version of Ramaswami’s formula. Commun. Statist. Stoch. Models 13, 223-238.

[13] Moustafa, M. D. (1957). Input-output Markov process. In Proc. Koninkl. Net. Akad. Wetensch. A60, pp. $112-118$.

[14] Neuts, M. F. (1981). Matrix-Geometric Solutions in Stochastic Models. An Algorithmic Approach. Johns Hopkins University Press, Baltimore, MD.

[15] Neuts, M. F. (1989). Structured Stochastic Matrices of M/G/1 Type and Their Applications. Marcel Dekker, New York.

[16] Neuts, M. F. (1994). An interesting random walk on the non-negative integers. J. Appl. Prob. 31, 48-58.

[17] Ramaswami, V. (1988). A stable recursion for the steady-state vector in Markov chains of M/G/1 type. Commun. Statist. Stoch. Models 4, 183-188.

[18] Ramaswami, V. (1997). Matrix analytic methods: a tutorial overview with some extensions and new results. In Matrix-Analytic Methods in Stochastic Models (Lecture Notes Pure Appl. Math. 183), Marcel Dekker, New York, pp. 261-296.

[19] Riska, A. (2002). Aggregate matrix-analytic techniques and their applications. Doctoral Thesis, The College of William and Mary, Williamsburg.

[20] ZhaO, Y. Q. (2000). Censoring technique in studying block-structured Markov chains. In Advances in Algorithmic Methods for Stochastic Models, Notable Publications, Neshanic Station, NJ, pp. 417-433. 